\title{
Kinetics of Step Propagation at the Sidewalls of 3D Islands and Nanowires*
}

\author{
Sergey N. Filimonov and Yuri Yu. Hervieu ${ }^{\dagger}$ \\ Department of Physics, Tomsk State University, Tomsk 634050, Russia \\ (Received 12 January 2014; Accepted 4 February 2014; Published 22 February 2014)
}

\begin{abstract}
Models of adatom surface diffusion and incorporation at the sidewalls of nanowires and 3D islands are proposed. The models account for the presence of an atomic step on the sidewall facet and for the adatom transport from the substrate to the sidewall. Peculiarities of the potential relief for migrating adatoms as well as the effect of step permeability are taken into account. It is shown that kinetics of the step propagation at the nanowire sidewall is affected by the presence of a strong sink for adatoms at the nanowire top facet and by adatom desorption from the sidewall. As a consequence, survival of the step at the nanowire sidewall depends strongly on the nanowire length. For 3D islands it is shown that the adatom concentration at the island apex can be considerable even in the absence of the deposition flux provided that the step is permeable for adatoms and there exists substantial material transfer from the wetting layer to the island facet. This favors formation of new steps at the island apex and may trigger the morphological transition of the 3D island. [DOI: 10.1380/ejssnt.2014.68]
\end{abstract}

Keywords: Models of surface kinetics; Epitaxy; Step formation and bunching; Surface diffusion; Nanowires

\section{INTRODUCTION}

It is well known that reproducible growth of atomically smooth crystal faces occurs by lateral motion of monatomic (elementary) steps $[1,2]$. At the same time grouping of the steps into step bunches may make the given crystal face unstable in respect to appearance of crystal facets of different orientations [3]. Nucleation and motion of elementary steps also plays an important role in the growth of nanoscale three-dimensional (3D) islands and nanowires. The radial growth of nanowires involves formation and propagation of monoatomic steps at atomically smooth nanowire sidewalls. These processes determine not only the radial growth rate of nanowires, but also stability of nanowires against shape transformations $[4,5]$. Similarly, the shape conserving growth of faceted 3D islands, such as hut-like 3D Ge islands on $\mathrm{Si}(001)$, is known to proceed by nucleation and propagation of elementary steps at the island facets, whereas bunching of the steps at the island facets is considered as the most plausible pathway for the experimentally observed shape transformation of the hut-clusters to dome-shaped 3D islands $[6,7]$.

In the present work we study theoretically the step dynamics at the sidewalls of 3D islands and nanowires with a simple one-dimensional (1D) surface diffusion model. By setting up the model we take into account peculiarities of the growth processes at the 3D island and nanowire sidewalls such as the presense of a strong sink for adatoms at the nanowire top, adatom supply from the substrate surface to the sidewall facets, and possibility for adatoms to cross the step edge at the sidewall without incorporation into the kink site (step permeability effect [8-10]). Using the developed model we show that survival of a step formed at the nanowire base depends strongly on the nanowire length. Since top of the nanowire is a strong sink for adatoms the step formed at the nanowire side-

\footnotetext{
*This paper was presented at the 12 th International Conference on Atomically Controlled Surfaces, Interfaces and Nanostructures (ACSIN-12) in conjunction with the 21st International Colloquium on Scanning Probe Microscopy (ICSPM21), Tsukuba International Congress Center, Tsukuba, Japan, November 4-8, 2013.

${ }^{\dagger}$ Corresponding author: ervye@mail.tsu.ru
}

wall is forced to decay in favor of the top facet. On the other hand the step formed at the base of a long nanowire might dissolve when direct adsorption at the sidewall is small as compared to desorption. At certain conditions a non-trivial re-entrant step behavior is expected.

In the case of $3 \mathrm{D}$ islands we show that adatom concentration close to the island apex might be substantial even if the deposition flux is switched off. This happens when the supersaturation on the wetting layer relatively to the step is high enough and the step is permeable for adatoms. When two steps are present at the island facet, the upper step may move faster than the lower one due to adatom transfer from the wetting layer to the upper step through the permeable lower step and due to partial dissolution of the lower step in favor of the upper one.

\section{STEP DYNAMICS AT THE SIDEWALL OF A NANOWIRE}

\section{A. Boundary value problem and step velocity}

Let us consider a regular array of identical cylindrical nanowires each growing within a circular "feeding" cell of a radius $R_{0}$ (Fig. 1(a)). Let at the given moment of time the nanowire has a radius $R$, length $L$ and contains an elementary step located at its sidewall at a distance $l$ from the substrate surface (Fig. 1(a)). Atoms are deposited on the surface with a flux $J$ at an angle $\alpha$ to the nanowire growth axis. Since the sidewall and the surrounding substrate surface represent different crystal faces, the adsorption energies and diffusion barriers for adatoms on the sidewall and on the substrate are, in general, different (Fig. 1(b)). Adatom concentrations on the substrate surface, $n_{s}$, and the nanowire sidewall, $n$, are assumed to obey the following steady state diffusion equations $[11,12]$

$$
\begin{gathered}
\frac{D_{s}}{r} \frac{d}{d r}\left(r \frac{d n_{s}}{d r}\right)+J \cos \alpha-\frac{n_{s}}{\tau_{s}}=0, \\
D \frac{d^{2} n}{d z^{2}}+J \sin \alpha-\frac{n}{\tau}=0
\end{gathered}
$$


(a)

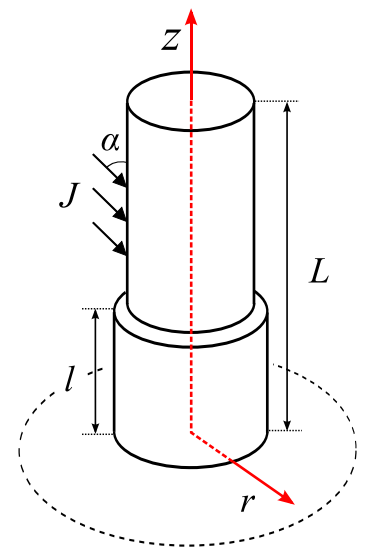

(b)

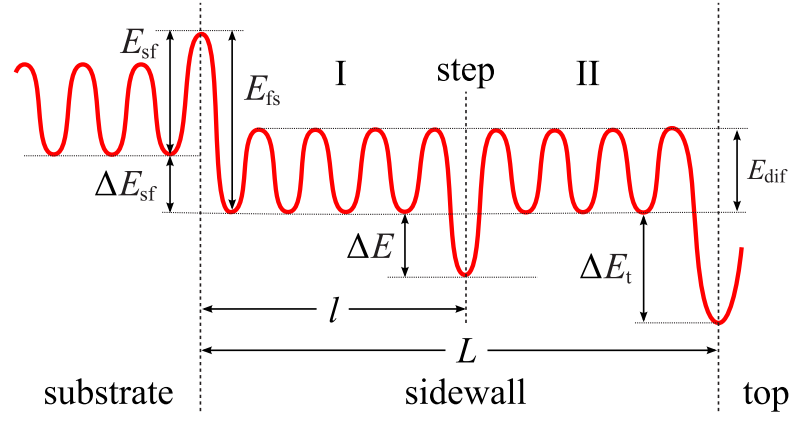

FIG. 1: (a) Cylindrical nanowire growing within a circular feeding area. (b) Potential energy landscape seen by an adatom migrating on the substrate surface and on the nanowire sidewall.

where $D_{s}$ and $D$ are the surface diffusion coefficients of adatoms, $\tau_{s}$ and $\tau$ are the lifetimes of adatoms (before desorption) on the substrate surface and on the sidewall facet, respectively. General solution of Eq. (1) and general solutions of Eq. (2) for the regions $I(0<z<l)$ and $I I$ $(l<z<L)$ has the form

$$
\begin{aligned}
n_{s}(r) & =J \tau_{s} \cos \alpha+c_{1} I_{0}\left(\frac{r}{\lambda_{s}}\right)+c_{2} K_{0}\left(\frac{r}{\lambda_{s}}\right), \\
n^{I}(z) & =J \tau \sin \alpha+a_{1} \cosh \left(\frac{z}{\lambda}\right)+b_{1} \sinh \left(\frac{z}{\lambda}\right), \\
n^{I I}(z) & =J \tau \sin \alpha+a_{2} \cosh \left(\frac{z-l}{\lambda}\right)+b_{2} \sinh \left(\frac{z-l}{\lambda}\right),
\end{aligned}
$$

where $I_{0}$ and $K_{0}$ are the modified Bessel functions of the first and second kind, respectively, $\lambda_{s}=\sqrt{D_{s} \tau_{s}}$ and $\lambda=\sqrt{D \tau}$ are the diffusion lengths of adatoms on the substrate surface and on the nanowire sidewall, respectively, $a_{i}, b_{i}$ and $c_{i}$ are six constants to be determined from the boundary conditions.

The boundary conditions at the line dividing the nanowire sidewall from the substrate surface follow from the balance equations for the surface diffusion fluxes and the fluxes of adatoms transferring from the substrate surface to the sidewall facet and vice versa:

$$
\begin{aligned}
\left.D_{s} \frac{d n_{s}}{d r}\right|_{r=R} & =k_{s f} n_{s}(R)-k_{f s} n^{I}(0), \\
\left.D \frac{d n^{I}}{d z}\right|_{z=0} & =k_{f s} n^{I}(0)-k_{s f} n_{s}(R) .
\end{aligned}
$$

Here $k_{s f}$ and $k_{f s}$ are the rate constants of the transition of adatoms from the substrate surface to the sidewall and from the sidewall to the substrate surface, respectively.
The zero flux condition is applied to the outer boundary of the "feeding" cell due to the symmetry of the problem:

$$
\left.\frac{d n_{s}}{d r}\right|_{r=R_{0}}=0 .
$$

The remaining three boundary conditions represent balance equations between the diffusion fluxes and the net fluxes of adatoms incorporating to the step from the upper $(-)$ and lower $(+)$ terraces, as well as to the top facet of the nanowire:

$$
\begin{aligned}
-\left.D \frac{d n^{I}}{d z}\right|_{z=l} & =\beta_{-}\left[n^{I}(l)-\tilde{n}\right], \\
\left.D \frac{d n^{I I}}{d z}\right|_{z=l} & =\beta_{+}\left[n^{I I}(l)-\tilde{n}\right], \\
-\left.D \frac{d n^{I I}}{d z}\right|_{z=L} & =k_{f t}\left[n^{I I}(L)-\widetilde{n}_{t}\right] .
\end{aligned}
$$

Here $\beta_{-}$and $\beta_{+}$are the incorporation coefficients of the step [2], $k_{f t}$ is the rate constant of the transition of adatoms from the sidewall to the top facet, $\widetilde{n}$ and $\tilde{n}_{t}$ are the concentrations of adatoms at the sidewall in equilibrium with the step and with the nanowire top facet, respectively.

All the lengths through the paper are expressed in the units of the surface lattice constant. It is also assumed that standard Arrhenius expressions hold for the rate constants $k_{\alpha \beta}=\nu \exp \left(-E_{\alpha \beta} / k_{B} T\right)$, where $E_{\alpha \beta}$ is the activation energy of the transition from $\alpha$ to $\beta, \nu$ is the attempt frequency, $k_{B}$ is the Boltzmann constant, $T$ is the substrate temperature. The equilibrium adatom concentrations are defined as $\tilde{n}=\exp \left(-\Delta E / k_{B} T\right)$ and $\tilde{n}_{t}=\exp \left(-\Delta E_{t} / k_{B} T\right)$, where $\Delta E$ and $\Delta E_{t}$ denote the energy gain due to incorporation of a sidewall adatom into the step and into the top nanowire facet, respectively (c.f. Fig. 1(b)).

From the solution of the boundary value problem (1)(8) we found the adatom incorporation flux into the step $g_{s t}=\beta_{-}\left[n^{I}(l)-\widetilde{n}\right]+\beta_{+}\left[n^{I I}(l)-\widetilde{n}\right]$, which is conveniently expressed as the linear combination of the relevant "driving forces":

$$
g_{s t}=\frac{D \widetilde{n}}{\lambda A_{s t} B_{s t}}\left(A_{s t} \sigma_{s}-B_{s t} \sigma_{t}+C_{s t} \sigma\right)
$$

Here $\sigma=J \tau \sin \alpha / \widetilde{n}-1$ is the supersaturation in the adlayer on the sidewall surface in respect to the step; $\sigma_{s}=J \tau_{s} \xi \cos \alpha / \widetilde{n}-1$ is the supersaturation in the adlayer on the substrate surface in respect to the step; $\sigma_{t}=1-\widetilde{n}_{t} / \widetilde{n}$ is the supersaturation at the step in respect to the nanowire top. The constant $\xi=k_{s f} / k_{f s}=$ $\exp \left(\Delta E_{s f} / k_{B} T\right)$ characterises the difference $\Delta E_{s f}$ between the adatom adsorption energy on the substrate surface and that on the sidewall of the nanowire (c.f. Fig. 1(b)). The constants $A_{s t}, B_{s t}$ and $C_{s t}$ are given by

$$
\begin{aligned}
A_{s t}= & \left(\Gamma_{+}+\Gamma_{t}\right) \cosh (\bar{L}-\bar{l})+\left(1+\Gamma_{+} \Gamma_{t}\right) \sinh (\bar{L}-\bar{l}) \\
B_{s t}= & \left(\Gamma_{-}+\Gamma_{s}\right) \cosh \bar{l}+\left(1+\Gamma_{-} \Gamma_{s}\right) \sinh \bar{l} \\
C_{s t}= & A_{s t}\left(\cosh \bar{l}+\Gamma_{s} \sinh \bar{l}-1\right)+ \\
& \quad+B_{s t}\left[\cosh (\bar{L}-\bar{l})+\Gamma_{t} \sinh (\bar{L}-\bar{l})-1\right]
\end{aligned}
$$


where $\bar{L}=L / \lambda ; \bar{l}=l / \lambda ; \Gamma_{ \pm}=D /\left(\beta_{ \pm} \lambda\right) ; \Gamma_{t}=D /\left(k_{f t} \lambda\right)$ and

$$
\Gamma_{s}=\frac{D}{k_{f s} \lambda}\left(1+\frac{k_{s f}}{D_{s}} \lambda_{s} G\right) .
$$

Here $G=-U\left(R / \lambda_{s}\right) / U^{\prime}\left(R / \lambda_{s}\right)$, where $U(x)=$ $K_{1}\left(R_{0} / \lambda_{s}\right) I_{0}(x)+I_{1}\left(R_{0} / \lambda_{s}\right) K_{0}(x)$ is a combination of modified Bessel functions and $U^{\prime}(x)$ is the derivative of $U(x)$ taken at $x=R / \lambda_{s}$.

The first term on the right hand side of (9) is the contribution of the adatom flux from the substrate surface. The second term is the exchange flux between the step and nanowire top. Finally, the third term in (9) describes the contribution of atoms adsorbing on the sidewall surface of the nanowire and incorporating into the step.

It should be noted that in the boundary conditions (6) and (7) we did not take the step permeability effect [8-10] into account. If every time there is a certain number of the kinks at the step edge the step permeability might be neglected when the mean distance between the kinks, $\lambda_{k}$, is smaller than the distance from the step edge to a competitive sink for adatoms, e.g. the distance to the top facet of the nanowire, $L-l$. In this case an adatom, after crossing the step edge, with a higher probability will come back to the kink than migrate to the top facet [13]. Evidently, the inequality $\lambda_{k}<L-l$ holds in the case of nanowires due to their high aspect ratio $L / R \gg 1$. Indeed, since the length of the step at the sidewall corresponds to the nanowire perimeter, then $\lambda_{k} \leq 2 \pi R \ll L-l$. Although this inequality breaks when the step edge is approaching top of the nanowire, for the most part of its lifetime the step at the nanowire sidewall can be considered as impermeable for adatoms.

\section{B. Critical nanowire length}

It is reasonable to assume that the top nanowire facet is a stronger sink for adatoms than the step at the nanowire sidewall. In this case $\widetilde{n}_{t}<\widetilde{n}$ and the supersaturation $\sigma_{t}$ lies in the range $(0 \ldots 1)$. Accordingly the exchange flux between the step and the top facet, described by the second term in the brackets on the right hand side of Eq. (9), is negative. This means that the step looses atoms in favor of the top nanowire facet. The supersaturations $\sigma$ and $\sigma_{s}$ may vary from -1 to infinity. Negative values of $\sigma$ correspond to small incidence angles $\alpha$ of the deposition flux $J$ onto the sidewall $(\sin \alpha<\widetilde{n} /(J \tau))$. In this case the adsorption layer at the sidewall surface is undersaturated in respect to the step and the third term in Eq. (9) is negative, indicating that the step dissolves due to detachments of atoms from the kinks and their subsequent desorption from the surface. These two effects slow down the step propagation rate and, as will be shown below, may even make the upward motion of the step to top of the nanowire impossible.

To determine the conditions under which the step will move upwards let us assume that there are no extra barriers for adatom attachment to the step edge and to the nanowire top. The corresponding potential energy surface is sketched in Fig. 1(b). In this case $\Gamma_{+}=\Gamma_{-}=\Gamma_{t}=$ $\Gamma=1 / \lambda$. Then it follows from Eq. (9) that at a given step position $l$ the flux $g_{s t}$ vanishes if the following equation holds:

$$
a s^{2}+b s+c=0 .
$$

Here $s=\exp (\bar{L}-\bar{l}), a=(1+\Gamma)(\sigma+(1+\Gamma) \delta), b=$ $-2\left(\sigma+\sigma_{t}\right), c=(1-\Gamma)(\sigma-(1-\Gamma) \delta)$ and

$$
\delta=\frac{\left(\Gamma_{s} \sinh \bar{l}+\cosh \bar{l}-1\right) \sigma+\sigma_{s}}{\left(1+\Gamma \Gamma_{s}\right) \sinh \bar{l}+\left(\Gamma+\Gamma_{s}\right) \cosh \bar{l}} .
$$

So, the step propagation rate becomes zero at the critical nanowire lengths given by $L_{1,2}^{*}=\lambda \ln s_{1,2}+l$, where $s_{1,2}$ are the two roots of Eq. (10).

Let us consider a step just formed at the nanowire base $(l \sim 1)$. Assuming $\lambda \gg 1(\Gamma \ll 1)$ we obtain the following approximated expressions for the coefficient $a$ and discriminant $\Delta=b^{2}-4 a c$ of the quadratic equation (10)

$$
a \approx \sigma+\frac{\sigma_{s}}{\kappa} ; \quad \Delta \approx 4\left[\left(2 \sigma+\sigma_{t}\right) \sigma_{t}+\frac{\sigma_{s}^{2}}{\kappa^{2}}\right],
$$

where $\kappa=(l+1) \Gamma+\Gamma_{s}$.

It should be noted that if $a>0\left(\sigma_{s} / \kappa>-\sigma\right)$, then $\left(2 \sigma+\sigma_{t}\right) \sigma_{t}+\left(\sigma_{s} / \kappa\right)^{2}>\left(\sigma+\sigma_{t}\right)^{2}>0$ and therefore discriminant $\Delta$ is also positive. This means that $g_{s t}>0$ when $s_{2}>s>-\infty$ or $\infty>s>s_{1}$. It can be shown that the second root $s_{2}<1$. The sign of the first root $s_{1}$ depends on the supersaturation $\sigma_{t}$ at the step in respect to the nanowire top. If top of the nanowire is a stronger sink for adatoms than the step $\left(\sigma_{t}>0\right)$, then $s_{1}>1$. If the relative supersaturation between the step and the top nanowire facet is zero $\left(\sigma_{t}=0\right)$ then $s_{1}=1$. Thus at $\sigma_{s} / \kappa>-\sigma$ there is a single critical length $L_{1}^{*}=\lambda \ln s_{1}+l$ and the step propagation toward the nanowire top is possible only at $L>L_{1}^{*}$.

If the incidence angle $\alpha$ of the deposition flux $J$ is small, it might happen that desorption of adatoms from the sidewall surface is not compensated by the adsorption. In this case the supersaturation $\sigma$ at the sidewall is negative. At $\sigma<0$ and at a positive but insufficiently high supersaturation on the substrate surface, $\sigma_{s}$, the inequality $\sigma_{s} / \kappa<-\sigma$ holds and the coefficient $a$ becomes negative. Here there are several possibilities.

If $\sigma<-\left[\sigma_{t}^{2}+\left(\sigma_{s} / \kappa\right)^{2}\right] /\left(2 \sigma_{t}\right)$, the discriminant $\Delta$ is negative and Eq. (10) has no real solutions. This means that $g_{s t}<0$ for any $s$, i.e. the step should dissolve at any nanowire length. In should be noted that the inequality $\Delta<0$ might be fulfilled only at a negative $\sigma$ and at a sufficiently large $\sigma_{t}$. Here simultaneous action of two factors, namely desorption of adatoms from the sidewall surface and the presence of a strong sink for adatoms at top of the nanowire, combined with insufficiently strong driving force for adatom transfer from the substrate surface to the step (small supersaturation $\sigma_{s}$ ) leads to dissolution of the step at any length of the nanowire.

If $0>\sigma>-\left[\sigma_{t}^{2}+\left(\sigma_{s} / \kappa\right)^{2}\right] /\left(2 \sigma_{t}\right)$, the discriminant $\Delta$ is positive. In this case $g_{s t}>0$ when $s_{1}>s>s_{2}$. It can be shown that both roots of the quadratic equation (10) are larger than unity if $\sigma_{t}+\sigma<0$. Then the step propagation toward the nanowire top is possible only in the limited range of the nanowire lengths: $L_{1}^{*}<L<L_{2}^{*}$. When $L<$ $L_{1}^{*}$ the step edge is too close to top of the nanowire and the step if forced to dissolve in favor of the top nanowire facet. 

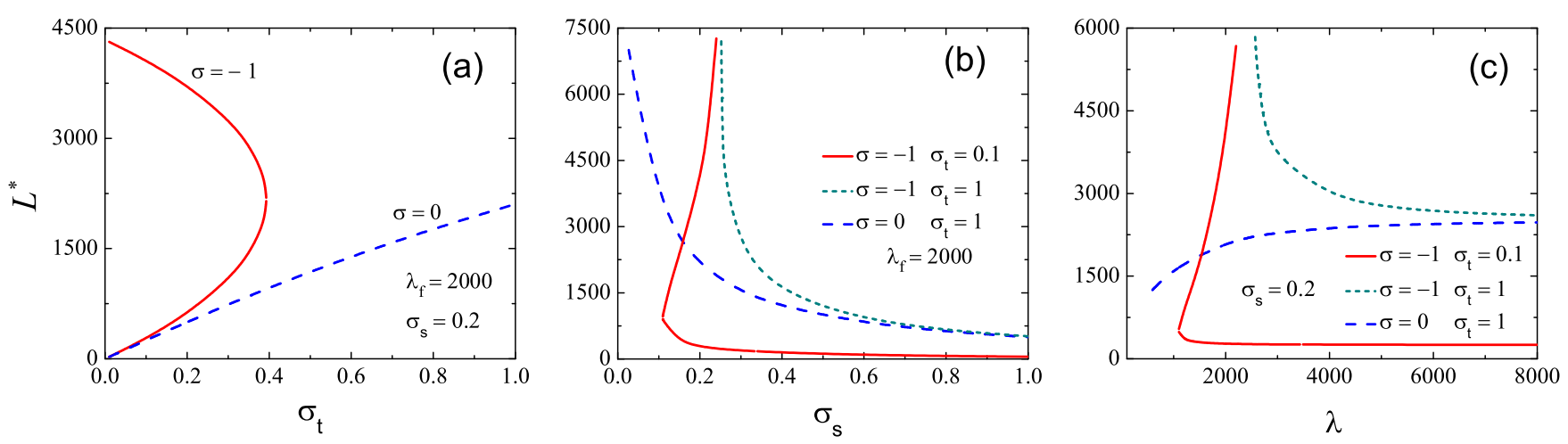

FIG. 2: Critical nanowire length as function of (a) the supersaturation at the step relatively to the nanowire top, (b) the supersaturation at the substrate surface relative to the step and (c) the adatom diffusion length on the sidewall.

With increasing nanowire length $L$ this effect weakens and in the interval $L_{1}^{*}<L<L_{2}^{*}$ the step moves upward by capturing adatoms transferred to the sidewall from the substrate surface. However, when the nanowire length exceeds the second critical length $L_{2}^{*}$, adatom supply from the substrate surface cannot compensate desorption from the sidewall surface leading to dissolution of the step.

Figure 2 shows dependencies of the critical nanowire length $L^{*}$ on (a) the supersaturation at the step relatively to the nanowire top, (b) the supersaturation at the substrate surface and (c) the adatom diffusion length on the sidewall calculated at $\Gamma_{s} / \Gamma=500$. In accordance with the discussion above, Fig. 2(a) demonstrates that in the case of normal incidence of the deposition flux onto the substrate $(\sigma=-1)$ and sufficiently high supersaturation at the step relatively to the nanowire top the step velocity should be negative irrespectively to the nanowire length. Similar situation takes place at $\sigma=-1$ and $\sigma_{t}>0$ when either the supersaturation on the substrate surface $\sigma_{s}$ or the diffusion length $\lambda$ is small (Fig. 2(b) and Fig. 2(c), respectively). In the case of moderate $\sigma_{t}$ (sufficiently high $\sigma_{s}$ or $\lambda$ ) step propagation toward the nanowire top is possible only in a limited range of the nanowire lengths. At $\sigma \geq 0$ the step moves upward if $L>L_{1}^{*}$, thereby $L_{1}^{*}$ increases with increasing $\sigma_{t}$ and $\lambda$ and decreases with increasing $\sigma_{s}$.

Let us now consider a step located far away from the substrate surface so that $l \gg \lambda$ (thereby $\bar{l} \gg 1$ ). Here as before the sign of $g_{s t}$ is determined by the solution of the quadratic equation (10), however the approximate expressions for the constant $a$ and discriminant $\Delta$ are different from (11). Indeed, applying $\bar{l} \gg 1$ to the exact expressions for the coefficients $a, b$ and $c$ one gets $a \approx 2 \sigma$ and $\Delta \approx 4\left(\sigma+\sigma_{t}\right)^{2}-16 \Gamma \sigma^{2}$. It should be noted that here $a$ and $\Delta$ are independent on the supersaturation on the substrate surface $\sigma_{s}$, because $l \gg \lambda$.

An analysis of possible solutions of Eq. (10) shows that in the case under consideration independent on the supersaturation $\sigma_{t}$ the step velocity is negative when $\sigma<0$. If $\sigma$ is positive, then at $\sigma_{t}<\Gamma \sigma$ the step velocity is positive for any $L-l$, while at $\sigma_{t}>\Gamma \sigma$ the step velocity is positive when $L-l>L_{1}^{*}-l=\lambda \ln s_{1}$. Since $\Gamma$ is typically much smaller unity, under moderate $\sigma$ and a nonzero supersaturation at the step relatively to the nanowire top, $\sigma_{t}$, the step cannot catch the top of the nanowire and after reaching the critical distance $L_{1}^{*}-l$ to the top it will move upward with the same velocity as the nanowire will elongate. This effect may trigger bunching of the steps propagating from the nanowire base. As a consequence a pensile-like shape of the nanowire may develop $[4,5]$.

\section{STEP PERMEABILITY EFFECT IN THE GROWTH OF FACETED 3D ISLANDS}

The growth mechanism of faceted 3D islands, such as pyramid-like $3 \mathrm{D}$ Ge hut-clusters on $\mathrm{Si}(001)$, is apparently similar to that of the radial growth of the nanowires. In both cases the sidewall facets are expanded by the nucleation and propagation of the elementary steps, however in the case of 3D islands the characteristic step length is comparable with the distance from the step to the island apex. Therefore even if every time there is a kink at the step edge, adatoms crossing the step edge without visiting the kink site at the step have good chances to reach the top of the facet and contribute to the nucleation and growth of new facet layers. So, in contrast to the case of nanowires, the influence of the step permeability effect on the growth kinetics of the 3D island facets should not be neglected. Moreover, in the case of the row-byrow mechanism of the step propagation, the step might be free of kinks during considerable fraction of time [14], so that the assumption of permanent presence of kinks at the step edge used throughout the given paper provides only a lower bound for the step permeability effect.

Similar to nanowires, bunching of the steps nucleated at the $3 \mathrm{D}$ island sidewall facets may result in a shape transformation of the $3 \mathrm{D}$ islands. For instance in $\mathrm{Ge} / \mathrm{Si}(001)$ heteroepitaxy the steps nucleate at the island apex and propagate to the island base. Nucleation of multiple steps at the (105) facets of Ge hut-clusters and their bunching trigger transformation of the hut-clusters to dome-shaped 3D islands [6]. Noticeably, at certain conditions the hut clusters may slowly grow in size even when the Ge flux is switched off [15]. Also the hut-to-dome transition of the island shape may occur both under Ge deposition and after annealing without Ge flux [6]. This points to the possibility that Ge atoms necessary for the nucleation and growth of new layers on the hut-cluster facets are provided by partial decomposition of the strained Ge wetting layer [15].

Here we study the step dynamics at the $3 \mathrm{D}$ island facets 


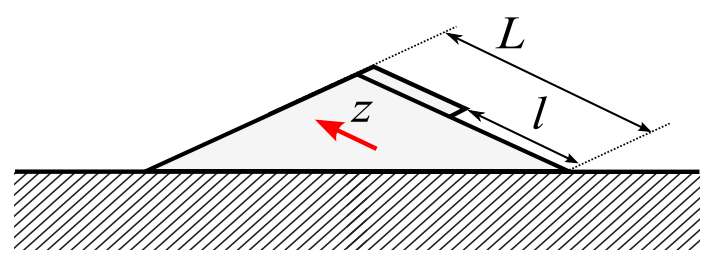

FIG. 3: Side-view of a pyramid-like 3D island.

using a simple $1 \mathrm{D}$ diffusion model similar to that used in the previous section. We generalize this model by taking into account the step permeability effect. Both adsorption of adatoms from the deposition flux and adatom supply from the wetting layer to the growing 3D island facet are considered. Geometry of the model is sketched in Fig. 3. We consider an atomically smooth facet of a 3D island containing a single elementary step nucleated at the facet apex and at the given moment of time located at a distance $l$ from the island base. Since at typical deposition conditions desorption of Ge adatoms from the surface is negligible we assume that concentration of adatoms on the $3 \mathrm{D}$ island facet satisfy the following continuity equation

$$
D \frac{d^{2} n}{d z^{2}}+J=0
$$

A general solution of (12) for the region $I$ in front of the step $(0<z<l)$ and region $I I$ behind the step $(l<z<L)$, contains four constants to be defined from the appropriate boundary conditions. The first boundary condition for the boundary between the island facet and the wetting layer represents the balance equation for the surface diffusion flux of adatoms and the resulting flux of adatoms transferring from the substrate surface to the facet:

$$
\left.D \frac{d n^{I}}{d z}\right|_{z=0}=k_{f s} n^{I}(0)-k_{s f} n_{s}
$$

This boundary condition is similar to that used in the previous section for the boundary between the nanowire sidewall and the substrate surface (Eq. (4)). However in the case of $3 \mathrm{D}$ islands we do not solve the surface diffusion problem for adatoms on the substrate surface but treat the adatom concentration on the substrate surface close to the $3 \mathrm{D}$ island, $n_{s}$, as an independent model parameter. Two other boundary conditions represent the balance equations between the fluxes of adatoms entering the step by surface diffusion and the fluxes of adatoms incorporating into the step and crossing the step without incorporation

$$
\begin{aligned}
& -\left.D \frac{d n^{I}}{d z}\right|_{z=l}=\beta_{+}\left[n^{I}(l)-\widetilde{n}\right]+\beta_{p}\left[n^{I}(l)-n^{I I}(l)\right] \\
& \left.D \frac{d n^{I I}}{d z}\right|_{z=l}=\beta_{-}\left[n^{I I}(l)-\tilde{n}\right]+\beta_{p}\left[n^{I I}(l)-n^{I}(l)\right]
\end{aligned}
$$

where $\beta_{p}$ is the step permeability coefficient [8-10]. The fourth boundary condition reflects the absence of the sink for adatoms at the facet apex

$$
\left.D \frac{d n^{I I}}{d z}\right|_{z=L}=0
$$

For simplicity the kink density at the step edge is assumed to be constant. In this case the kinetic coefficients $\beta_{ \pm}$and the step permeability coefficient $\beta_{p}$ do not depend on the adatom concentration $n$ and Eqs.(12)-(16) represent a linear boundary value problem which can be solved exactly.

From the solution of Eqs. (12)-(16) one obtains the following expression for the adatom concentration at the facet apex $(z=L)$

$$
n^{I I}(L)=\frac{J(L-l)^{2}}{2 D}+l_{-} \frac{\left[\left(l_{+}+l_{s}+l\right)(L-l)+\eta_{+}\left(L l_{s}+L l-\frac{l^{2}}{2}\right)\right] \frac{J}{D}+\eta_{+}\left(\xi n_{s}-\widetilde{n}\right)}{\left(1+\eta_{-}\right) l_{+}+\left(1+\eta_{-}+\eta_{+}\right)\left(l_{s}+l\right)}+\widetilde{n}
$$

where $\xi=k_{s f} / k_{f s}, \eta_{ \pm}=\beta_{p} / \beta_{ \pm}, l_{ \pm}=D / \beta_{ \pm}$, and $l_{s}=D / k_{f s}$. It follows from (17) that when a step is present at the $3 \mathrm{D}$ island facet, there are three channels for adatom supply to the island apex: (i) adsorption from the molecular beam, (ii) transfer of adatoms from the wetting layer via the permeable step, and (iii) detachment of adatoms from the kink sites at the step edge.

Equation (17) is simplified when the barrier for adatom incorporation into the step from the lower terrace equals to the surface diffusion barrier $E_{d i f}$, while adatom at- taching to the step edge from the upper terrace has to overcome the barrier $E_{d i f}+\Delta E_{E S}$, where $\Delta E_{E S}$ is the Ehrlich-Schwoebel barrier [16, 17]. Using expressions for the kinetic coefficients derived in [10] it can be shown that $\eta_{-}=\eta_{+} l_{E S}$ and $l_{-}=l_{+} l_{E S}$, where $l_{E S}=$ $\exp \left(\Delta E_{E S} / k_{B} T\right)$ is the characteristic length related to the Ehrlich-Schwoebel barrier. Moreover $\eta_{-}+\eta_{+}=2 \eta=$ $l_{+}-1$, where $\eta$ is the permeability parameter of the step at the absence of the Ehrlich-Schwoebel effect, i.e. at $\Delta E_{E S}=0$. Then, assuming $l \gg 1$, one obtains

$$
n^{I I}(L)=\frac{\left[\left(\eta_{-}+l_{s}+l\right)(L-l)\left(L-l+2 l_{E S}\right)+\eta_{-} L\left(2 l_{s}+L\right)\right] \frac{J}{2 D}+\eta_{-}\left(\xi n_{s}-\tilde{n}\right)}{\eta_{-}+l_{s}+l}+\tilde{n} .
$$


According to (18), adatom concentration at the 3D island apex increases with increasing step permeability (with increasing $\eta_{-}$). Thereby even at zero deposition flux $J=0$ the value of $n^{I I}(L)$ might be high and might substantially exceed the equilibrium adatom concentration $\widetilde{n}$. This happens when the supersaturation on the wetting layer surface relatively to the step is so high that

$$
\sigma_{s}^{\prime} \equiv \frac{\xi n_{s}}{\widetilde{n}}-1 \gg \frac{\eta_{-}+l_{s}+l}{\eta_{-}} .
$$

Moreover even at a non-zero deposition flux adatom concentration at the island apex $n^{I I}(L)$ is determined mainly by the adatom transfer from the wetting layer if in addition to (19) the second term in the numerator on the left hand side of Eq. (18) is greater than the first one. The step permeability effect and a deep trap for adatoms at the island facet $(\xi \gg 1)$ act in favor of this inequality. On the contrary, the Ehrlich-Schwoebel effect hinders the material transfer from the wetting layer surface to the 3D island apex.

Using the solution of the boundary value problem (12)(16) one may calculate the step propagation velocity $V_{s t}=$ $\beta_{+}\left[n^{I}(l)-\widetilde{n}\right]+\beta_{-}\left[n^{I I}(l)-\widetilde{n}\right]$. Assuming $l \gg 1$, one has

$$
V_{s t}=\frac{J\left[2 L\left(l_{s}+l\right)-l^{2}\right]+2 D \tilde{n} \sigma_{s}^{\prime}}{2\left(\eta_{-}+l_{s}+l\right)} .
$$

It follows from (20), that $V_{s t}$ decreases with increasing step permeability. If

$$
\xi n_{s}-\widetilde{n} \gg J\left[\frac{L}{k_{f s}}+\frac{l(2 L-l)}{2 D}\right],
$$

the step velocity is determined mainly by the adatom supply from the wetting layer. Inequality (21) demonstrates that the presence of a deeper trap for adatoms at the island facet relatively to the wetting layer surface reinforces the role of the adatom transfer from the wetting layer.

Apart from the transition to the multilayer growth of the 3D island facets, a necessary condition for the transformation of the hut-cluster to the dome-island is the bunching of the steps formed at its facets. In the following we analyze possible mechanisms of the step bunching on the 3D island facet using two steps as an example. The presence of the second step is taken into account by replacing Eq. (16) in the boundary value problem (12)(16) by the balance equation for the adatom diffusion flux and flux of adatoms incorporating into the second (upper) step

$$
\left.D \frac{d n^{I I}}{d z}\right|_{z=l_{2}}=k_{f t}\left[n\left(l_{2}\right)-\widetilde{n}_{t}\right] .
$$

Here $l_{2}$ is the distance from the island base to the upper step, $\widetilde{n}_{t}$ is the adatom concentration on the facet corresponding to the equilibrium with the upper step. For simplicity the upper step was assumed to be impermeable for adatoms. We also assumed that due to better strain relaxation at the island apex the upper step is a stronger sink for adatoms than the lower step, i.e. $\widetilde{n}_{t}<\widetilde{n}$.

From the solution of the modified boundary value problem we obtained the following expression for the velocity of the lower step

$$
V_{1}=\frac{D \widetilde{n}\left[\left(l_{E S}+\Delta l\right) \sigma_{s}^{\prime}-\left(l_{s}+l_{1}\right) \sigma_{t}^{\prime}\right]+C_{1} J / 2}{\left(l_{s}+l_{1}\right)\left(l_{E S}+\Delta l\right)+\eta_{-}\left(l_{E S}+l_{s}+l_{2}\right)},
$$

where $C_{1}=\left(l_{s}+l_{1}\right) \Delta l^{2}+\left(l_{E S}+\Delta l\right)\left(2 l_{s}+l_{1}\right) l_{1}$ and $\sigma_{t}^{\prime}=$ $1-\widetilde{n}_{t} / \tilde{n}$. Here $l_{1}$ is the distance from the island base to the lower step and $\Delta l=l_{2}-l_{1}$ is the width of the terrace between the upper and the lower steps. Deriving (23) we assumed that $l_{1}, \Delta l \gg 1$. For the velocity of the upper step one has

$$
V_{2}=\frac{D \widetilde{n}\left[\eta_{-} \sigma_{s}^{\prime}+\left(l_{s}+l_{1}+\eta_{-}\right) \sigma_{t}^{\prime}\right]+C_{2} J / 2}{\left(l_{s}+l\right)\left(l_{E S}+\Delta l\right)+\eta_{-}\left(l_{E S}+l_{s}+l_{2}\right)}+J\left(L-l_{2}\right),
$$

where $C_{2}=\left(2 l_{E S}+\Delta l\right)\left(l_{s}+l_{1}\right) \Delta l+\eta_{-}\left(2 l_{s} l_{2}+l_{1}^{2}+\left(2 l_{E S}+\right.\right.$ $\Delta l) \Delta l$ ). The second term on the right hand side of (24) reflects the contribution of the deposition of adatoms on the terrace behind the upper step.

It follows from the obtained expressions that convergence of the steps $\left(V_{2}>V_{1}\right)$ is favored by the EhrlichSchwoebel barrier $\left(l_{E S} \gg 1\right)$, by a deeper trap for atoms at the kink site at the upper step than that at the lower step $\left(\widetilde{n} \gg \widetilde{n}_{t}\right)$ and by the permeability of the lower step (by a high value of the permeability parameter $\eta_{-}$). It is essential that even at zero deposition flux pairing of the steps may occur without dissolution of the lower step provided that substantial adatom transfer from the wetting layer is present. Indeed putting $J=0$ at (23) one finds that $V_{1}>0$ if

$$
\sigma_{s}^{\prime}>\frac{l_{s}+l_{1}}{l_{E S}+\Delta l} \sigma_{t}^{\prime} .
$$

Under this condition the inequality $V_{2}>V_{1}$ is carried out if

$$
\sigma_{s}^{\prime}<\frac{2\left(l_{s}+l_{1}\right)+\eta_{-}}{l_{E S}+\Delta l-\eta_{-}} \sigma_{t}^{\prime}
$$

which presumes that $\eta_{-}<l_{E S}+\Delta l$. If the step permeability parameter is so high that $\eta_{-}>l_{E S}+\Delta l$, then $V_{2}>V_{1}$ independent on the supersaturation $\sigma_{s}^{\prime}$.

\section{CONCLUSION}

We have studied theoretically adatom diffusion and step dynamics at the sidewalls of nanowires and at the facets of $3 \mathrm{D}$ islands. It has been shown that survival of the elementary steps at the nanowire sidewalls depends strongly on the deposition conditions and on the nanowire length. In the case of short nanowires the presence of a strong sink for adatoms at top of the nanowire leads to dissolution of the step formed at the nanowire sidewall if the loss of atoms by the step in favor of the top facet is not compensated by the adatom transfer from the substrate surface. When the nanowire length exceeds certain critical value, the step propagation from the nanowire base toward the nanowire top becomes possible. Thereby the step nucleated at the base of a long nanowire may also be forced to dissolve if desorption of adatoms from the sidewall surface outbalances adsorption of adatoms on the sidewall (for instance in the case of normal incidence of the deposition flux onto the substrate). Thus, under certain conditions, the radial growth of a nanowire by nucleation and propagation of steps at its sidewall is possible only in a limited range of the nanowire lengths.

It has been shown that adatom concentration close to the apex of a $3 \mathrm{D}$ island might be substantially higher than 
the equilibrium adatom concentration even if the deposition flux is zero. This happens when the supersaturation on the wetting layer surface relatively to the step is high and the step is permeable for adatoms. Thereby the step moves mainly by capturing adatoms detaching from the metastable wetting layer. When two steps are present at the island facet, the upper step being a stronger sink for adatoms may move faster than the lower one by consuming adatoms both supplied by the partially dissolving lower step and adatoms detached from the wetting layer and transferred to the upper step through the permeable lower step.

\section{Acknowledgments}

This study is supported by by the Russian Foundation for Basic Research (grants No. 13-02-01097 and 13-02$12160)$.
[1] W. K. Burton, N. Cabrera, and F. Frank, Philos. Trans. R. Soc. London, Ser. A 243, 299 (1951).

[2] I. Markov, Crystal Growth for Beginners, 2nd ed. (World Scientific, Singapore, 2003).

[3] K. Yagi, H. Minoda, and M. Degawa, Surf. Sci. Rep. 43, 45 (2001).

[4] C. Chen, M. C. Plante, C. Fradin, and R. R. LaPierre, J. Mater. Res. 21, 2801 (2006).

[5] M. C. Plante and R. R. LaPierre, J. Cryst. Growth 310 356 (2008).

[6] F. Montalenti, et al., Phys. Rev. Lett. 93, 216102 (2004).

[7] S. Cereda and F. Montalenti, Phys. Rev. B 75, 195321 (2007).

[8] M. Ozdemir and A. Zangwill, Phys. Rev. B 45, 3718 (1992).

[9] O. Pierre-Louis, Phys. Rev. Lett. 87, 106104 (2001).
[10] S. N. Filimonov and Yu. Yu. Hervieu, Surf. Sci. 553, 133 (2004).

[11] V. G. Dubrovskii, et al., Semiconductors 40, 1075 (2006).

[12] V. G. Dubrovskii, et al., Phys. Rev. B 79, 205316 (2009).

[13] S. N. Filimonov and Yu. Yu. Hervieu, Russian Microelectronics 40, 52 (2011).

[14] S. N. Filimonov and Yu. Yu. Hervieu, Int. J. Nanosci. 6, 237 (2007).

[15] M. R. McKay, J. A. Venables, and J. Drucker, Phys. Rev. Lett. 101, 216104 (2008).

[16] G. Ehrlich and F. G. Hudda, J. Chem. Phys. 44, 1039 (1966).

[17] R. L. Schwoebel and E. J. Shipsey, J. Appl. Phys. 37, 3682 (1966). 